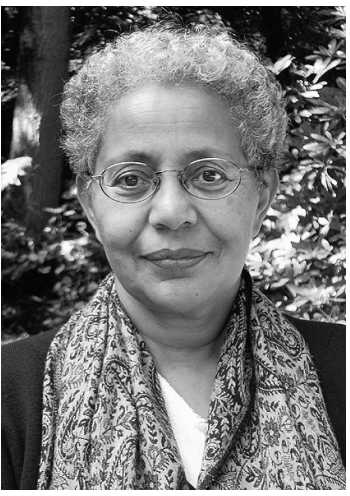

\title{
Politics and Homeopathy
}

There are things in our world that, when they happen, will upset most of us. The horror of floods, earthquakes, war, crime, rape, child abuse, terrorist attacks etc. is something we share and no one in his or her right mind would want any of these to happen to anybody.

Then there are things that we share, as a nation, a group, or a family, that are more specific to that particular group; not every group will feel the same about these issues, because they all relate to age, place, race, culture, religion.

In the last few years a term has been introduced that describes a kind of thinking or attitude that is considered done or not done; it's called 'political correctness'. I do not know where the term originates, or who created it, but I do know that it's used frequently.

The interesting thing is that it seems to be clearly understood by the users what the difference is between correct and incorrect. People even use it as a way of allowing themselves to say something that otherwise would have been unacceptable within their group, age or cultural identity. In other words, specific conditions create different 'characteristics' and change the 'modalities'.

What is characteristic about the French and what modality is the desire to drink wine with their meal? Or what is characteristic about the Ferrums and what is the modality to get nauseous while eating? Is pathology always pathology, worldwide, culturally indifferent and religiously irrelevant? Would the desire to drink wine with her meal for a strict Muslim woman, or an aversion to drink wine with his meal for the Frenchman, be signs of pathology? Is 'falling in love with one of her own sex' in Italy today pathology and was the sexual experimentation with someone of one's own gender in ancient Roman culture a sign of health and normality?

I remember a patient of mine who kept on saying that she needed to become more loving and tolerant of her husband's rude behaviour and asked me if I could remedy this problem she was suffering from. When I asked her to describe her husband's behaviour, she described constant moodiness, rudeness, lack of responsibility and indifference towards his family. When I was so bold as to tell her that there isn't a remedy to help her to live happily with maltreatment, she sat quietly for some time and said, 'then I have a problem'. I replied, 'yes, I think you have' and then we both sat quietly for some time.

The need for open-mindedness and the invitation to look beyond the obvious, not to take everything for granted or automatically accept authority, regardless of its intelligence or moral standards, are not only necessary for the homeopath but also - and especially - for our world, a world which frequently, regularly and periodically seems to go insane.

We have not much else to rely on in dealing with bombing, killing, flooding, raping, political lying, religious fanaticism etc.

I was shocked to hear that a deeply religious group in Israel was actually praying for the destruction of their political leader, but I can understand their feelings.

I was moved to tears by the conflict and the refusal of soldiers in the same country to forcefully remove their own people from their land and homes, after they had protected them for so many years. I understand their despair and struggle and regard it as a hopeful sign. Nevertheless I understand that they are ordered to do this.

Is this editorial becoming too political? I don't know. It might be, but can you always tell the difference between homeopathy and politics? I can't.

Human suffering is present everywhere, all the time, in many forms and variations. Political decisions (also in our homes) are being made frequently, with huge consequences. In our practice we get to hear the stories of these consequences. How individually do we suffer from pathology?

How on a worldwide scale do we need to be treated?

It seems to be an ongoing quest. If this issue of Links can contribute to supporting our colleagues in their homeopathic journey, it has fulfilled its purpose.

Corrie Hiwat, editor 